University of South Florida

DIGITAL COMMONS

Digital Commons @ University of

@ UNIVERSITY OF SOUTH FLORIDA

South Florida

$1-1-2016$

\title{
2016 Work Plan USF St. Petersburg
}

USF

Follow this and additional works at: https://digitalcommons.usf.edu/usf_accountability_reports

\section{Scholar Commons Citation}

USF, "2016 Work Plan USF St. Petersburg" (2016). USF Accountability Reports. 62.

https://digitalcommons.usf.edu/usf_accountability_reports/62

This Article is brought to you for free and open access by the USF Archives at Digital Commons @ University of South Florida. It has been accepted for inclusion in USF Accountability Reports by an authorized administrator of Digital Commons @ University of South Florida. For more information, please contact digitalcommons@usf.edu. 


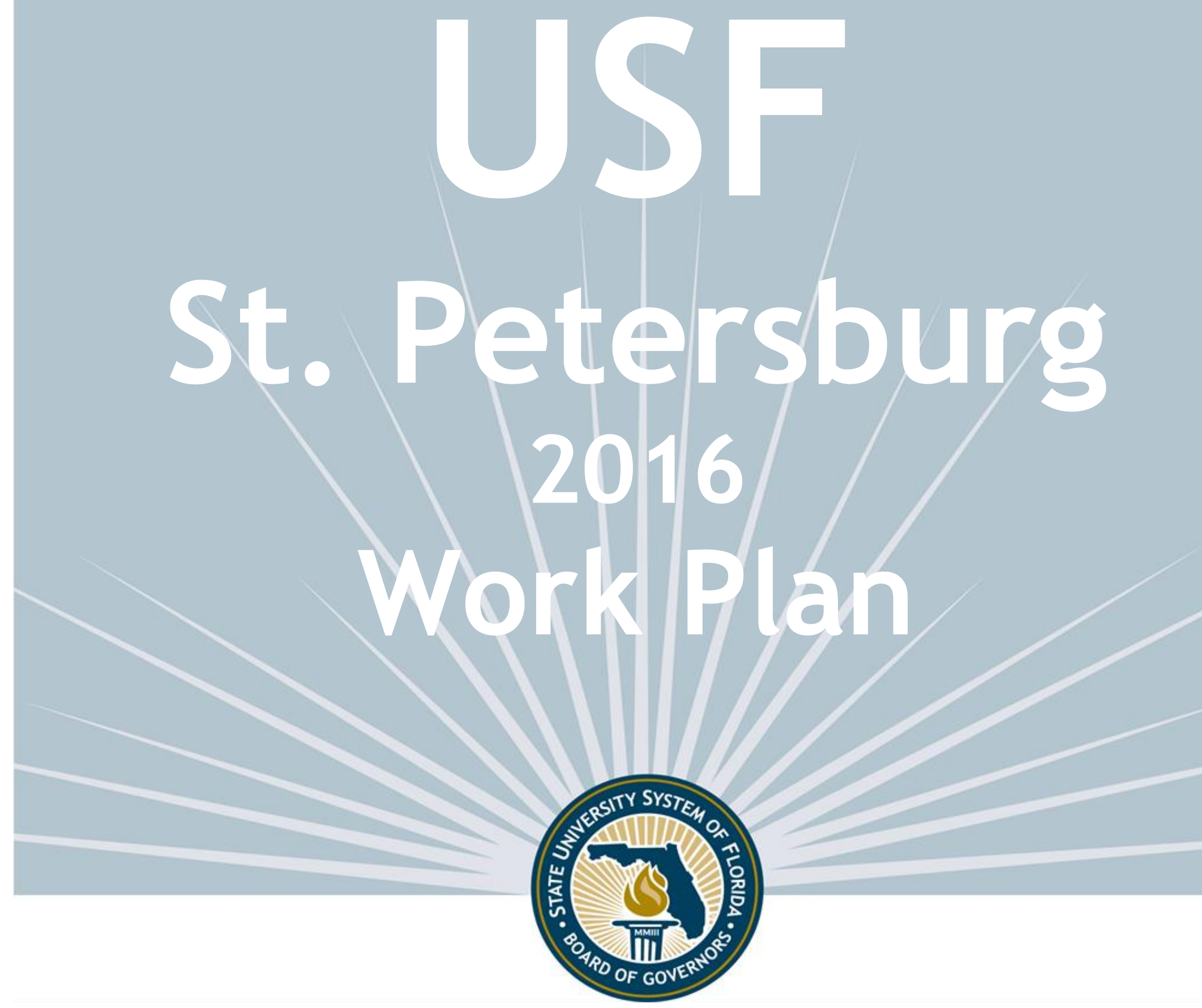

University of South Florida-Saint Petersburg

University Work Plan Presentation

for Board of Governors June 2016 Meeting

STATE UNIVERSITY SYSTEM of FLORIDA $\mid$ Board of Governors 


\section{INTRODUCTION}

The State University System of Florida has developed three tools that aid in guiding the System's future.

1) The Board of Governors' 2025 System Strategic Plan is driven by goals and associated metrics that stake out where the System is headed;

2) The Board's Annual Accountability Report provides yearly tracking for how the System is progressing toward its goals;

3) Institutional Work Plans connect the two and create an opportunity for greater dialogue relative to how each institution contributes to the System's overall vision.

These three documents assist the Board with strategic planning and with setting short-, mid- and long-term goals. The Board will use these documents to help advocate for all System institutions and foster even greater coordination with the institutions and their Boards of Trustees.

Longer-term goals will inform future agendas of the Board's Strategic Planning Committee. The Board's acceptance of a work plan does not constitute approval of any particular component, nor does it supersede any necessary approval processes that may be required for each component. 


\section{TABLE OF CONTENTS}

\section{STRATEGY}
a. Mission Statement
b. Vision Statement
c. Statement of Strategy
d. Strengths and Opportunities
e. Key Initiatives \& Investments

2. PERFORMANCE BASED FUNDING METRICS

3. KEY PERFORMANCE INDICATORS

a. Teaching \& Learning

b. Institution Specific Goals

\section{ENROLLMENT PLANNING}

5. ACADEMIC PROGRAM COORDINATION

6. STUDENT DEBT \& NET COST

\section{UNIVERSITY REVENUES}

8. TUITION, FEES AND HOUSING PROJECTIONS

\section{DEFINITIONS}




\section{MISSION STATEMENT (What is your purpose?)}

The mission of USFSP is to inspire scholars to lead lives of impact. As an integral and complementary part of the multi-institutional USF System, The University of South Florida St. Petersburg (USFSP) reflects a distinctive identity and mission while contributing to and benefiting from the association, cooperation, and shared resources of a premier national research university.

\section{VISION STATEMENT (What do you aspire to?)}

USF St. Petersburg will shine. USFSP faculty and administrators will work shoulder-to-shoulder with students and community partners to build a better world. We will challenge ourselves to excel in research, teaching and service. USFSP will be a premier urban institution recognized for its vibrant community of scholars who engage and improve the community.

\section{STATEMENT OF STRATEGY (How will you get there?)}

Given your mission, vision, strengths and available resources, provide a brief description of your market and your strategy for addressing and leading it.

Guided by the USF System's strategic goals and USFSP's mission and vision, our strategies are also informed by Vision 20/20 and the ongoing USFSP strategic plan implementation process for 2014 - 2019. To achieve the goal of supporting a distinctive identity as a top teaching and research institution, USFSP will emphasize faculty scholarship and research, student performance and discovery, campus culture, and strategic partnerships. USFSP will pursue these goals by providing the organizational infrastructure and the sustainable funding to make this possible. Throughout the process, USFSP's top priority is student success through increased student retention and graduation rates. Vision 20/20 articulates our six strategic goals for the future:

- Distinctive Identity

- Student Success and Culture

- Faculty Excellence in Teaching and Research

- Strategic Partnerships

- Infrastructure to Meet Current and Future Needs

- Sustainable Funding

Included in the strategic plan is the goal to increase annual enrollment with students served rising to 10,000 students by 2025 . This increase represents growth in the annual students served as measured by the total number of students taking credit hours in a year at USFSP (Fall 2015 unduplicated headcount was 4,749 USFSP students, as shown on page 11's Enrollment Planning Tables). This initiative will help stakeholders appreciate how planned growth will stabilize and energize USFSP and the USF System. 


\section{STRENGTHS AND OPPORTUNITIES (within 3 years)}

\section{What are your core capabilities, opportunities and challenges for improvement?}

USFSP's core capabilities include excellence in teaching using all forms of delivery, high levels of faculty-student interaction, a vibrant research culture, an entrepreneurial spirit, strong and collaborative community engagement and partnerships, as well as data-based decision-making. USFSP provides students with an intimate intellectual environment to facilitate their growth as active learners and instill life-long habits of the mind.

USFSP's strengths and opportunities include its distinguished faculty, who, along with staff and administrators, are highly committed to student success. Another strength is its location. Being in the heart of St. Petersburg's culture and arts corridor, its premier health facilities and the business district (and newly developing Innovation District) opens up rich educational opportunities for students, as well as for employees of these companies who seek coursework and degrees on our campus.

Moreover, our programs continue to accumulate prestigious recognitions and accreditations. Also, we are Pinellas County's only public research institution, and we benefit from being a valued member of the USF System.

Our strong and growing community partnerships provide us with an opportunity to secure additional external input and build civic engagement. By developing appropriate support structures, our distinguished faculty will be able to enhance teaching and further their research, including externally funding for their research through grants and contracts.

As we strive to grow our student population, we are balancing regional workforce needs, applicant interest and campus support to continually evolve a future-oriented palette of programs, degrees and services. This goal requires us to actively recruit students who reflect our regional diversity as well as out-of-state and international students who bring global diversity to our campus. In addition, it entails instituting retention programs to ensure that students who start at USFSP will graduate from USFSP in a timely fashion, prepared to earn more advanced degrees or to enter the workforce.

We are also a young institution that is still developing its identity and the infrastructure necessary to fully realize our potential. Although we see many advantages of being an urban institution, there are limited opportunities for physical expansion. As our STEM programs prosper, we will have growing demands for teaching and laboratory space, as well as financial aid for our student population. 


\section{KEY INITIATIVES \& INVESTMENTS (within 3 years)}

Describe your top three key initiatives for the next three years that will drive improvement in Academic Quality, Operational Efficiency, and Return on Investment.

1. Develop and implement focused initiatives for greater student retention and graduation: Strategic Plan implementation efforts resulted in a proposal to launch a comprehensive first-year experience program titled Compass. Compass is designed to integrate new students into the academic and socio-cultural communities of USFSP. Activities target first-year student needs, success strategies and peer coaching. Compass upper-division peer coaches help guide first-year students to be successfully involved on campus, in the classroom and with faculty. Preparation for the launch took place during Spring 2015 and the implementation of this cutting-edge program was initiated in Summer 2015. We continue to review closely the "lessons learned" from the Quality Enhancement Plan (QEP) - all in an effort to address retention from all fronts. It is expected that these efforts will improve student retention rates, 4- and 6-year graduation rates as well as help reduce excess credit hours at USFSP.

\section{Develop and implement focused initiatives to improve faculty excellence:}

Strategic Plan implementation efforts resulted in proposals to increase faculty diversity as well as excellence in research and teaching. Focused recruitment and incentives have led to significantly increased faculty diversity for hires among Hispanic and African American faculty who will join USFSP beginning in Fall 2016. In addition, a minority post-doc program begun Spring 2016 is aimed at recruiting and mentoring minority faculty over the next few years. A new internal grant proposal program is designed to provide support for junior faculty to be mentored by senior experienced faculty members from outside the university to assist them with publication and grant-writing experience. A new Center for Innovative Teaching and Learning was launched in Spring 2016 and will begin to provide instructional assistance to faculty in Fall 2016.

3. Provide the requisite physical, human and technological infrastructure for USFSP to sustain its current areas of excellence and to grow by design:

USFSP will enhance current facilities to support current and future programs and faculty and student needs, including classrooms, laboratories, studios and a new student service corridor. Technology upgrades were completed for all classrooms in Spring 2016 and four experimental classrooms were introduced as prototypes for classroom upgrades in Fall 2016. We will assess and improve infrastructure for career development, internships, partnerships, community engagement, and the experience for freshman, sophomore, and transfer students. 


\section{PERFORMANCE BASED FUNDING METRICS}

\begin{tabular}{|c|c|c|c|c|c|c|}
\hline & $\begin{array}{r}2015 \\
\text { ACTUAL } \\
\end{array}$ & $\begin{array}{c}2016 \\
\text { ACTUAL }\end{array}$ & $\begin{array}{c}2017 \\
\text { GOALS }\end{array}$ & $\begin{array}{c}2018 \\
\text { GOALS }\end{array}$ & $\begin{array}{c}2019 \\
\text { GOALS }\end{array}$ & $\begin{array}{l}2020 \\
\text { GOALS }\end{array}$ \\
\hline $\begin{array}{l}\text { Percent of Bachelor's Graduates } \\
\text { Enrolled or Employed }(\mathbf{\$ 2 5 , 0 0 0 + )} \\
\text { within the U.S. One Year After } \\
\text { Graduation }\end{array}$ & $\begin{array}{c}61.1 \% \\
2012-13\end{array}$ & $\begin{array}{c}67.1 \% \\
2013-14\end{array}$ & $\begin{array}{l}69.0 \% \\
2014-15\end{array}$ & $\begin{array}{l}71.0 \% \\
2015-16\end{array}$ & $\begin{array}{l}73.0 \% \\
2016-17\end{array}$ & $\begin{array}{l}76.0 \% \\
2017-18\end{array}$ \\
\hline $\begin{array}{l}\text { Median Wages of Bachelor's } \\
\text { Graduates Employed Full-time } \\
\text { in Florida One-Year After Graduation }\end{array}$ & $\begin{array}{c}\$ 34,800 \\
2012-13\end{array}$ & $\underset{2013-14}{\$ 35,400}$ & $\underset{2014-15}{\$ 36,000}$ & $\underset{2015-16}{\$ 36,300}$ & $\underset{2016-17}{\$ 37,200}$ & $\underset{2017-18}{\$ 37,800}$ \\
\hline $\begin{array}{l}\text { Cost per Bachelor's Degree } \\
\text { Costs to the University }\end{array}$ & \multicolumn{6}{|c|}{ Data reported at the USF System level only. } \\
\hline $\begin{array}{l}\text { FTIC } 6 \text { year Graduation Rate * } \\
\text { for full- and part-time students }\end{array}$ & $\begin{array}{c}31.6 \%^{1} \\
2008-14\end{array}$ & $\begin{array}{c}38.3 \%^{2} \\
2009-15\end{array}$ & $\begin{array}{c}42.0 \% \\
2010-16\end{array}$ & $\begin{array}{c}44.0 \% \\
2011-17\end{array}$ & $\begin{array}{l}45.0 \% \\
2012-18\end{array}$ & $\begin{array}{c}50.0 \% \\
2013-19\end{array}$ \\
\hline $\begin{array}{l}\text { Academic Progress Rate } * \\
\text { FTIC } 2 \text { year Retention Rate with GPA>2 }\end{array}$ & $\underset{2013-14}{61.6 \%}{ }^{3}$ & $\begin{array}{c}66.9 \% \\
2014-15\end{array}$ & $\begin{array}{c}68.0 \% \\
2015-16\end{array}$ & $\begin{array}{c}72.0 \% \\
2016-17\end{array}$ & $\begin{array}{l}74.0 \% \\
2017-18\end{array}$ & $\begin{array}{c}76.0 \% \\
2018-19\end{array}$ \\
\hline $\begin{array}{l}\text { Bachelor's Degrees Awarded } \\
\text { Within Programs of Strategic } \\
\text { Emphasis }\end{array}$ & $\begin{array}{c}36.5 \% \\
2013-14\end{array}$ & $\begin{array}{l}42.3 \% \\
2014-15\end{array}$ & $\begin{array}{l}43.0 \% \\
2015-16\end{array}$ & $\begin{array}{l}43.0 \% \\
2016-17\end{array}$ & $\begin{array}{l}43.0 \% \\
2017-18\end{array}$ & $\begin{array}{c}45.0 \% \\
2018-19\end{array}$ \\
\hline $\begin{array}{l}\text { University Access Rate } \\
\text { Percent of Fall Undergraduates } \\
\text { with a Pell grant }\end{array}$ & $\begin{array}{l}40.0 \% \\
\text { Fall } 2013\end{array}$ & $\begin{array}{l}41.6 \% \\
\text { Fall } 2014\end{array}$ & $\begin{array}{l}40.0 \% \\
\text { Fall } 2015\end{array}$ & $\begin{array}{l}40.0 \% \\
\text { Fall } 2016\end{array}$ & $\begin{array}{l}40.0 \% \\
\text { Fall } 2017\end{array}$ & $\begin{array}{l}40.0 \% \\
\text { Fall } 2018\end{array}$ \\
\hline $\begin{array}{l}\text { Graduate Degrees Awarded } \\
\text { Within Programs of Strategic } \\
\text { Emphasis }\end{array}$ & $\begin{array}{l}33.5 \% \\
2013-14\end{array}$ & $\begin{array}{l}29.7 \% \\
2014-15\end{array}$ & $\begin{array}{c}30.2 \% \\
2015-16\end{array}$ & $\begin{array}{c}30.2 \% \\
2016-17\end{array}$ & $\begin{array}{l}30.0 \% \\
2017-18\end{array}$ & $\begin{array}{c}30.2 \% \\
2018-19\end{array}$ \\
\hline $\begin{array}{l}\text { BOG METRIC: } \\
\text { Percent of Bachelor's Degrees } \\
\text { Without Excess Hours }\end{array}$ & $\begin{array}{c}67.8 \% \\
2013-14\end{array}$ & $\begin{array}{c}69.5 \% \\
2014-15\end{array}$ & $\begin{array}{c}70.0 \% \\
2015-16\end{array}$ & $\begin{array}{c}72.0 \% \\
2016-17\end{array}$ & $\begin{array}{c}74.0 \% \\
2017-18\end{array}$ & $\begin{array}{c}76.0 \% \\
2018-19\end{array}$ \\
\hline $\begin{array}{l}\text { UBOT METRIC: } \\
\text { Number of Post-doctoral } \\
\text { Appointees }\end{array}$ & $\begin{array}{c}0 \\
\text { Fall } 2012\end{array}$ & $\begin{array}{c}0 \\
\text { Fall } 2013\end{array}$ & $\begin{array}{c}0 \\
\text { Fall } 2014\end{array}$ & $\begin{array}{c}0 \\
\text { Fall } 2015\end{array}$ & $\begin{array}{c}0 \\
\text { Fall } 2016\end{array}$ & $\begin{array}{c}2 \\
\text { Fall } 2017\end{array}$ \\
\hline
\end{tabular}

Note: Metrics are defined in appendix. For more information about the PBF model visit: $h$ ttp://www.flbog.edu/about/budget/performance funding.php.

${ }^{*} \mathrm{FTIC}$ retention/academic progress rate and 6 -Year graduation rate percentages represent students starting and ending at USFSP.

$152.6 \%$ of USFSP students of the 2008 cohort graduated within the USF System

$260.3 \%$ of USFSP students of the 2009 cohort graduated within the USF System

$374.2 \%$ of USFSP students of the 2013 cohort were retained within the USF System

${ }^{4} 76.3 \%$ of USFSP students of the 2014 cohort were retained within the USF System 
Teaching \& Learning Metrics (from 2025 System Strategic Plan that are not included in PBF or Preeminence)

\begin{tabular}{|c|c|c|c|c|c|c|}
\hline & $\begin{array}{c}2015 \\
\text { ACTUAL }\end{array}$ & $\begin{array}{c}2016 \\
\text { ACTUAL }\end{array}$ & $\begin{array}{l}2017 \\
\text { GOALS }\end{array}$ & $\begin{array}{c}2018 \\
\text { GOALS }\end{array}$ & $\begin{array}{c}2019 \\
\text { GOALS }\end{array}$ & $\begin{array}{l}2020 \\
\text { GOALS }\end{array}$ \\
\hline $\begin{array}{l}\text { 2. Freshmen in Top } 10 \% \\
\text { of Graduating High School Class }\end{array}$ & $\begin{array}{l}15.0 \% \\
\text { Fall } 2014\end{array}$ & $\begin{array}{l}12.0 \% \\
\text { Fall } 2015\end{array}$ & $\begin{array}{l}12.0 \% \\
\text { Fall } 2016\end{array}$ & $\begin{array}{l}14.0 \% \\
\text { Fall } 2017\end{array}$ & $\begin{array}{l}16.0 \% \\
\text { Fall } 2018\end{array}$ & $\begin{array}{c}18.0 \% \\
\text { Fall } 2019\end{array}$ \\
\hline $\begin{array}{l}\text { 4. Time to Degree } \\
\text { for FTICs in } 120 h \text { programs }\end{array}$ & $\begin{array}{c}4.5 \\
2013-14\end{array}$ & $\begin{array}{c}4.1 \\
2014-15\end{array}$ & $\begin{array}{c}4.3 \\
2015-16\end{array}$ & $\begin{array}{c}4.3 \\
2016-17\end{array}$ & $\begin{array}{c}4.3 \\
2017-18\end{array}$ & $\begin{array}{c}4.3 \\
2018-19\end{array}$ \\
\hline $\begin{array}{l}\text { 5. Four-Year FTIC } \\
\text { Graduation Rates * } \\
\text { full- and part-time students }\end{array}$ & $\underset{2010-14}{21.0 \%}{ }^{1}$ & $\underset{2011-15}{21.0 \%}{ }^{2}$ & $\begin{array}{c}26.0 \% \\
2012-16\end{array}$ & $\begin{array}{c}26.0 \% \\
2013-17\end{array}$ & $\begin{array}{c}31.0 \% \\
2014-18\end{array}$ & $\begin{array}{c}33.0 \% \\
2015-19\end{array}$ \\
\hline $\begin{array}{l}\text { 8. Bachelor's Degrees Awarded } \\
\text { First Majors Only }\end{array}$ & $\begin{array}{c}821 \\
2013-14\end{array}$ & $\begin{array}{l}825 \\
2014-15\end{array}$ & $\begin{array}{l}886 \\
2015-16\end{array}$ & $\begin{array}{c}913 \\
2016-17\end{array}$ & $\begin{array}{c}928 \\
2017-18\end{array}$ & $\begin{array}{c}943 \\
2018-19\end{array}$ \\
\hline $\begin{array}{l}\text { 9. Graduate Degrees Awarded } \\
\text { First Majors Only }\end{array}$ & $\begin{array}{c}167 \\
2013-14\end{array}$ & $\begin{array}{c}222 \\
2014-15\end{array}$ & $\begin{array}{c}225 \\
2015-16\end{array}$ & $\begin{array}{c}225 \\
2016-17\end{array}$ & $\begin{array}{c}230 \\
2017-18\end{array}$ & $\begin{array}{c}235 \\
2018-19\end{array}$ \\
\hline $\begin{array}{l}\text { 10. Bachelor's Degrees Awarded } \\
\text { to African-American \& Hispanic } \\
\text { Students }\end{array}$ & $\begin{array}{c}16.0 \% \\
2013-14\end{array}$ & $\begin{array}{l}18.0 \% \\
2014-15\end{array}$ & $\begin{array}{l}20.0 \% \\
2015-16\end{array}$ & $\begin{array}{l}22.0 \% \\
2016-17\end{array}$ & $\begin{array}{l}24.0 \% \\
2017-18\end{array}$ & $\begin{array}{c}26.0 \% \\
2018-19\end{array}$ \\
\hline $\begin{array}{l}\text { 11. Adult (Aged } 25+\text { ) } \\
\text { Undergraduates Enrolled }\end{array}$ & $\begin{array}{l}33.0 \% \\
\text { Fall } 2013\end{array}$ & $\begin{array}{l}33.0 \% \\
\text { Fall } 2014\end{array}$ & $\begin{array}{l}33.0 \% \\
\text { Fall } 2015\end{array}$ & $\begin{array}{l}33.0 \% \\
\text { Fall } 2016\end{array}$ & $\begin{array}{l}30.0 \% \\
\text { Fall } 2018\end{array}$ & $\begin{array}{l}28.0 \% \\
\text { Fall } 2019\end{array}$ \\
\hline $\begin{array}{l}\text { 12. Percent of Undergraduate } \\
\text { FTE in Online Courses }\end{array}$ & $\begin{array}{c}32.0 \% \\
2013-14\end{array}$ & $\begin{array}{c}32.0 \% \\
2014-15\end{array}$ & $\begin{array}{c}32.0 \% \\
2015-16\end{array}$ & $\begin{array}{l}32.0 \% \\
2016-17\end{array}$ & $\begin{array}{c}32.0 \% \\
2017-18\end{array}$ & $\begin{array}{c}32.0 \% \\
2018-19\end{array}$ \\
\hline $\begin{array}{l}\text { 16. Percent of Bachelor's } \\
\text { Degrees in STEM \& Health }\end{array}$ & $\begin{array}{l}9.0 \% \\
2013-14\end{array}$ & $\begin{array}{c}15.0 \% \\
2014-15\end{array}$ & $\begin{array}{c}19.0 \% \\
2015-16\end{array}$ & $\begin{array}{c}21.0 \% \\
2016-17\end{array}$ & $\begin{array}{c}24.0 \% \\
2017-18\end{array}$ & $\begin{array}{c}26.0 \% \\
2018-19\end{array}$ \\
\hline $\begin{array}{l}\text { 18. Percent of Graduate Degrees } \\
\text { in STEM \& Health }\end{array}$ & $\begin{array}{l}2.0 \% \\
2013-14\end{array}$ & $\begin{array}{l}1.0 \% \\
2014-15\end{array}$ & $\begin{array}{l}2.2 \% \\
2015-16\end{array}$ & $\begin{array}{l}2.2 \% \\
2016-17\end{array}$ & $\begin{array}{l}3.0 \% \\
2017-18\end{array}$ & $\begin{array}{l}5.1 \% \\
2018-19\end{array}$ \\
\hline IMPROVING METRICS & & $\begin{array}{c}5 \\
\text { of } 10\end{array}$ & $\begin{array}{c}6 \\
\text { of } 10\end{array}$ & $\begin{array}{c}4 \\
\text { of } 10\end{array}$ & $\begin{array}{c}8 \\
\text { of } 10\end{array}$ & $\begin{array}{c}8 \\
\text { of } 10\end{array}$ \\
\hline
\end{tabular}

${ }^{*} \mathrm{FTIC} 4-$ Year graduation rate percentages represent students starting and ending at USFSP.

$130 \%$ of USFSP students of the 2010 cohort graduated within the USF System

$233 \%$ of USFSP students of the 2011 cohort graduated within the USF System 


\section{KEY PERFORMANCE INDICATORS (continued)}

Institution Specific Goals (optional)

To further distinguish the university's distinctive mission, the university may choose to provide additional narrative and metric goals that are based on the university's own strategic plan.

Narrative Goals. Goal 1. Improve baccalaureate retention and graduation. USFSP will focus on: 1) improving student outcomes in key mathematics courses through continued implementation of its Quality Enhancement Plan; 2) improving access to academic support(i.e. tutoring); 3) strengthening academic advising and university-wide interventions for increasing student success

\begin{tabular}{|c|c|c|c|c|c|c|}
\hline & $\begin{array}{c}2015 \\
\text { ACTUAL }\end{array}$ & $\begin{array}{c}2016 \\
\text { ACTUAL }\end{array}$ & $\begin{array}{l}2017 \\
\text { GOALS }\end{array}$ & $\begin{array}{c}2018 \\
\text { GOALS }\end{array}$ & $\begin{array}{c}2019 \\
\text { GOALS }\end{array}$ & $\begin{array}{l}2020 \\
\text { GOALS }\end{array}$ \\
\hline $\begin{array}{l}\text { Bachelor's Degree in Areas of } \\
\text { Strategic Emphasis }\end{array}$ & 309 & 349 & 360 & 370 & 390 & 400 \\
\hline $\begin{array}{l}\text { Percent of Course Sections Offered } \\
\text { via Distance and Blended Learning }\end{array}$ & $18 \%$ & $22 \%$ & $25 \%$ & $26 \%$ & $26 \%$ & $26 \%$ \\
\hline $\begin{array}{l}\text { Maintain Carnegie Community } \\
\text { Engagement Classification }\end{array}$ & Yes & Yes & Yes & Yes & Yes & Yes \\
\hline
\end{tabular}




\section{ENROLLMENT PLANNING}

Planned Headcount Enrollment by Student Type (for all students at all campuses)

\begin{tabular}{|c|c|c|c|c|c|c|c|}
\hline & $\begin{array}{c}\text { FALL } 2013 \\
\text { ACTUAL }\end{array}$ & $\begin{array}{c}\text { FALL } 2014 \\
\text { ACTUAL }\end{array}$ & $\begin{array}{c}\text { FALL } 2015 \\
\text { ACTUAL }\end{array}$ & $\begin{array}{c}\text { FALL } 2016 \\
\text { PLAN }\end{array}$ & $\begin{array}{c}\text { FALL } 2017 \\
\text { PLAN }\end{array}$ & $\begin{array}{c}\text { FALL } 2018 \\
\text { PLAN }\end{array}$ & $\begin{array}{c}\text { FALL } 2019 \\
\text { PLAN }\end{array}$ \\
\hline \multicolumn{8}{|l|}{ UNDERGRADUATE } \\
\hline FTIC & 1,636 & 1,518 & 1,609 & 1,704 & 1,807 & 1,897 & 1,992 \\
\hline AA Transfers ${ }^{1}$ & 1,446 & 1,427 & 1,464 & 1,511 & 1,586 & 1,665 & 1,748 \\
\hline Other ${ }^{2}$ & 868 & 890 & 872 & 926 & 972 & 1,023 & 1,074 \\
\hline Subtotal & 3,950 & 3,835 & 3,945 & 4,141 & 4,365 & 4,585 & 4,814 \\
\hline \multicolumn{8}{|l|}{ GRADUATE $^{3}$} \\
\hline Master's & 552 & 530 & 540 & 562 & 584 & 607 & 631 \\
\hline Research Doctoral & 0 & 0 & 0 & 0 & 0 & 0 & 0 \\
\hline Professional Doctoral & 0 & 0 & 0 & 0 & 0 & 0 & 0 \\
\hline Subtotal & 552 & 530 & 540 & 562 & 584 & 607 & 631 \\
\hline \multicolumn{8}{|l|}{ UNCLASSIFIED } \\
\hline H.S. Dual Enrolled & 0 & 0 & 0 & 0 & 0 & 0 & 0 \\
\hline Other ${ }^{4}$ & 238 & 231 & 264 & 275 & 286 & 297 & 308 \\
\hline Subtotal & 238 & 231 & 264 & 275 & 286 & 297 & 308 \\
\hline TOTAL & 4,740 & 4,596 & 4,749 & 4,978 & 5,235 & 5,489 & 5,753 \\
\hline
\end{tabular}

Notes: This table reports the number of students enrolled at the university by student type categories. The determination for undergraduate, graduate and unclassified is based on the institutional class level values. Unclassified refers to a student who has not yet been formally admitted into a degree program but is enrolled. The student type for undergraduates is based on the Type of Student at Time of Most Recent Admission. The student type for graduates is based on the degree that is sought and the student CIP code. (1) Includes AA Transfers from the Florida College System. (2) Undergraduate - Other includes Post-Baccalaureates who are seeking a degree. (3) Includes Medical students. (4) Unclassified - Other includes Post-Baccalaureates who are not seeking a degree.

\section{Planned FTE Enrollment by Method of Instruction (for all students at all campuses)}

\begin{tabular}{|c|c|c|c|c|c|c|c|}
\hline & $\begin{array}{l}2012-13 \\
\text { ACTUAL }\end{array}$ & $\begin{array}{l}2013-14 \\
\text { ACTUAL }\end{array}$ & $\begin{array}{l}\text { 2014-15 } \\
\text { ACTUAL }\end{array}$ & $\begin{array}{l}2015-16 \\
\text { PLAN }\end{array}$ & $\begin{array}{l}2016-17 \\
\text { PLAN }\end{array}$ & $\begin{array}{l}2017-18 \\
\text { PLAN }\end{array}$ & $\begin{array}{l}2018-19 \\
\text { PLAN }\end{array}$ \\
\hline \multicolumn{8}{|l|}{ UNDERGRADUATE } \\
\hline Distance (80-100\%) & 1,347 & 1,228 & 1,172 & 1,179 & 1,226 & 1,275 & 1,326 \\
\hline Hybrid (50-79\%) & 37 & 67 & 82 & 39 & 39 & 40 & 40 \\
\hline Traditional (0-50\%) & 2,688 & 2,537 & 2,386 & 2,460 & 2584 & 2,688 & 2,798 \\
\hline $\begin{array}{r}\text { Subtotal } \\
\text { GRADUATE }\end{array}$ & 4,072 & 3,832 & 3,640 & 3,678 & 3,850 & 4,003 & 4,164 \\
\hline Distance (80-100\%) & 154 & 183 & 203 & 203 & 211 & 220 & 228 \\
\hline Hybrid (50-79\%) & 0 & 4 & 19 & 3 & 3 & 3 & 3 \\
\hline Traditional (0-50\%) & 234 & 242 & 219 & 235 & 252 & 262 & 273 \\
\hline Subtotal & 387 & 428 & 441 & 441 & 466 & 485 & 504 \\
\hline
\end{tabular}

Note: Full-time Equivalent (FTE) student is a measure of instructional activity that is based on the number of credit hours that students enroll. FTE is based on the standard national definition, which divides undergraduate credit hours by 30 and graduate credit hours by 24 . Distance Learning is a course in which at least 80 percent of the direct instruction of the course is delivered using some form of technology when the student and instructor are separated by time or space, or both (per $1009.24(17)$, F.S.). Hybrid is a course where $50 \%$ to $79 \%$ of the instruction is delivered using some form of technology, when the student and instructor are separated by time or space, or both (per SUDS data element 2052). Traditional refers to primarily face to face instruction utilizing some form of technology for delivery of supplemental course materials for no more than $49 \%$ of instruction (per SUDS data element 2052).

Data Provided by USF at the institution level 


\section{ENROLLMENT PLANNING (continued)}

\section{Planned FTE Enrollment Plan by Student Level}

\begin{tabular}{|c|c|c|c|c|c|c|c|c|}
\hline & $\begin{array}{l}\text { 2014-15 } \\
\text { ACTUAL }\end{array}$ & $\begin{array}{c}2015-16 \\
\text { ESTIMATE }\end{array}$ & $\begin{array}{c}2016-17 \\
\text { PLAN }\end{array}$ & $\begin{array}{c}2017-18 \\
\text { PLAN }\end{array}$ & $\begin{array}{l}\text { 2018-19 } \\
\text { PLAN }\end{array}$ & $\begin{array}{c}2019-20 \\
\text { PLAN }\end{array}$ & $\begin{array}{l}2020-21 \\
\text { PLAN }\end{array}$ & $\begin{array}{c}2021-22 \\
\text { PLAN }\end{array}$ \\
\hline \multicolumn{9}{|c|}{ STATE FUNDABLE } \\
\hline \multicolumn{9}{|c|}{ RESIDENT } \\
\hline LOWER & 1,190 & 1,293 & 1,358 & 1,424 & 1,484 & 1,512 & 1,573 & 1,636 \\
\hline UPPER & 2,253 & 2,154 & 2,264 & 2,355 & 2,447 & 2,520 & 2,621 & 2,726 \\
\hline GRAD I & 420 & 416 & 432 & 450 & 468 & 486 & 506 & 526 \\
\hline GRAD ॥ & 0 & 0 & 0 & 0 & 0 & 0 & 0 & 0 \\
\hline TOTAL & 3,863 & 3,863 & 4,054 & 4,229 & 4,399 & 4,519 & 4,700 & 4,887 \\
\hline
\end{tabular}

\section{NON RESIDENT}

\begin{tabular}{lrrrrrrrrr}
\hline LOWER & 49 & 62 & 64 & 67 & 70 & 72 & 75 & 78 & $4.0 \%$ \\
UPPER & 66 & 80 & 83 & 86 & 89 & 93 & 97 & 101 & $4.0 \%$ \\
GRAD I & 13 & 16 & 17 & 17 & 18 & 19 & 19 & 20 & $4.0 \%$ \\
GRAD II & 0 & 0 & 0 & 0 & 0 & 0 & 0 & 0 & N/A \\
\hline TOTAL & $\mathbf{1 2 8}$ & $\mathbf{1 5 7}$ & $\mathbf{1 6 4}$ & $\mathbf{1 7 0}$ & $\mathbf{1 7 7}$ & $\mathbf{1 8 4}$ & $\mathbf{1 9 1}$ & $\mathbf{1 9 9}$ & $\mathbf{4 . 0 \%}$
\end{tabular}

TOTAL

\begin{tabular}{|c|c|c|c|c|c|c|c|c|c|}
\hline LOWER & 1,240 & 1,355 & 1,422 & 1,491 & 1,554 & 1,585 & 1,648 & 1,714 & $3.8 \%$ \\
\hline UPPER & 2,319 & 2,234 & 2,347 & 2,441 & 2,536 & 2,613 & 2,718 & 2,826 & $3.8 \%$ \\
\hline GRAD I & 432 & 432 & 449 & 467 & 486 & 505 & 525 & 546 & $4.0 \%$ \\
\hline GRAD II & 0 & 0 & 0 & 0 & 0 & 0 & 0 & 0 & $\mathrm{~N} / \mathrm{A}$ \\
\hline TOTAL & 3,991 & 4,020 & 4,218 & 4,399 & 4,576 & 4,703 & 4,891 & 5,087 & $3.8 \%$ \\
\hline \multicolumn{10}{|c|}{ NOT STATE FUNDABLE } \\
\hline LOWER & 31 & 37 & 38 & 39 & 41 & 43 & 44 & 46 & $3.7 \%$ \\
\hline UPPER & 50 & 54 & 40 & 42 & 43 & 45 & 47 & 49 & $4.1 \%$ \\
\hline GRAD I & 8 & 9 & 8 & 8 & 8 & 9 & 9 & 9 & $2.4 \%$ \\
\hline GRAD II & 0 & 0 & 0 & 0 & 0 & 0 & 0 & 0 & $\mathrm{~N} / \mathrm{A}$ \\
\hline TOTAL & 89 & 99 & 86 & 89 & 92 & 97 & 100 & 104 & $3.8 \%$ \\
\hline
\end{tabular}

Note: Full-time Equivalent (FTE) student is a measure of instructional activity that is based on the number of credit hours that students enroll. FTE is based on the standard national definition, which divides undergraduate credit hours by 30 and graduate credit hours by 24 . Note*: The Planned Annual Growth Rate is a compounded rate based on the following formula: (2021-22 value divided by the 2016-17 value) to the (1/5) exponent minus one.

Data Provided by USF at the institution level 
FINAL - JUNE 2016

\section{ACADEMIC PROGRAM COORDINATION}

New Programs For Consideration by University in AY 2016-17

The S.U.S. Council of Academic Vice Presidents (CAVP) Academic Program Coordination Work Group will review these programs as part of their on-going coordination efforts. The programs listed below are based on the 2015 Work Plan list for programs under consideration for 2016-17.

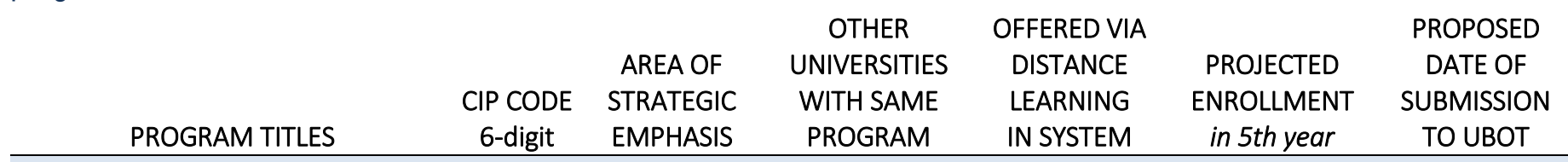

BACHELOR'S PROGRAMS

MASTER'S, SPECIALIST AND OTHER ADVANCED MASTER'S PROGRAMS

\begin{tabular}{lllllll}
\hline MS Conservation Biology & 26.1307 & STEM & UCF & $0 \%$ & 30 & Spring 2017
\end{tabular}

\section{DOCTORAL PROGRAMS}

New Programs For Consideration by University in 2017-19

These programs will be used in the 2017 Work Plan list for programs under consideration for 2017-18.

\begin{tabular}{|c|c|c|c|c|c|c|}
\hline & & & OTHER & OFFERED VIA & & PROPOSED \\
\hline & & AREA OF & UNIVERSITIES & DISTANCE & PROJECTED & DATE OF \\
\hline & CIP CODE & STRATEGIC & WITH SAME & LEARNING & ENROLLMENT & SUBMISSION \\
\hline PROGRAM TITLES & 6-digit & EMPHASIS & PROGRAM & IN SYSTEM & in 5 th year & TO UBOT \\
\hline BACHELOR'S PROGRAMS & & & & & & \\
\hline
\end{tabular}

MASTER'S, SPECIALIST AND OTHER ADVANCED MASTER'S PROGRAMS 


\section{STUDENT DEBT \& NET COST}

\section{Student Debt Summary}

\begin{tabular}{lccccc} 
& $2010-11$ & $2011-12$ & $2012-13$ & $2013-14$ & $2014-15$ \\
\hline Percent of Bachelor's Recipients with Debt & $48 \%$ & $48 \%$ & $55 \%$ & $51 \%$ & $56 \%$ \\
\hline $\begin{array}{l}\text { Average Amount of Debt } \\
\text { for Bachelor's who have graduated with debt }\end{array}$ & $\$ 22,840$ & $\$ 24,100$ & $\$ 19,860$ & $\$ 22,160$ & $\$ 19,705$ \\
& & & & & $2012-15$ \\
NSLDS Cohort Year & $2008-11$ & $2009-12$ & $2010-13$ & $2011-14$ & Preliminary \\
\hline Student Loan Cohort Default Rate (3rd Year) & $10.1 \%$ & $9.0 \%$ & $7.5 \%$ & $5.4 \%$ & $5.2 \%$
\end{tabular}

Cost of Attendance (for Full-Time Undergraduate Florida Residents in the Fall and Spring of 2015-16)

\begin{tabular}{ccccccc} 
& $\begin{array}{c}\text { TUITION } \\
\& \text { FEES }\end{array}$ & $\begin{array}{c}\text { BOOKS } \& \\
\text { SUPPLIES }\end{array}$ & $\begin{array}{c}\text { ROOM } \\
\& \text { BOARD }\end{array}$ & TRANSPORTATION & $\begin{array}{c}\text { OTHER } \\
\text { EXPENSES }\end{array}$ & TOTAL \\
\hline ON-CAMPUS & $\$ 5,822$ & $\$ 1,200$ & $\$ 9,400$ & $\$ 1,600$ & $\$ 2,500$ & $\$ 20,522$ \\
\hline AT HOME & $\$ 5,822$ & $\$ 1,200$ & $\$ 4,700$ & $\$ 1,600$ & $\$ 2,500$ & $\$ 15,822$ \\
\hline
\end{tabular}

Estimated Net Cost by Family Income (for Full-Time Undergraduate Florida Residents in the Fall and Spring of 2015-16)

\begin{tabular}{|c|c|c|c|c|c|c|}
\hline \multirow{2}{*}{$\begin{array}{l}\text { FAMILY } \\
\text { INCOME } \\
\text { GROUPS }\end{array}$} & \multicolumn{2}{|c|}{ FULL-TIME RESIDENT } & \multirow{2}{*}{$\begin{array}{c}\text { AVG. NET } \\
\text { COST OF } \\
\text { ATTENDANCE }\end{array}$} & \multirow{2}{*}{$\begin{array}{c}\text { AVG. NET } \\
\text { TUITION } \\
\& \text { FEES }\end{array}$} & \multirow{2}{*}{$\begin{array}{c}\text { AVG. } \\
\text { GIFT AID } \\
\text { AMOUNT }\end{array}$} & \multirow{2}{*}{$\begin{array}{c}\text { AVG. } \\
\text { LOAN } \\
\text { AMOUNT }\end{array}$} \\
\hline & HEADCOUNT & 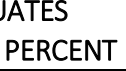 & & & & \\
\hline Below $\$ 40,000$ & 662 & $35 \%$ & $\$ 12,099$ & $-\$ 2,420$ & $\$ 7,641$ & $\$ 4,541$ \\
\hline$\$ 40,000-\$ 59,999$ & 240 & $13 \%$ & $\$ 13,496$ & $-\$ 712$ & $\$ 5,942$ & $\$ 3,795$ \\
\hline$\$ 60,000-\$ 79,999$ & 194 & $10 \%$ & $\$ 15,675$ & $\$ 1,711$ & $\$ 3,642$ & $\$ 4,763$ \\
\hline$\$ 80,000-\$ 99,999$ & 132 & $7 \%$ & $\$ 16,677$ & $\$ 3,168$ & $\$ 2,216$ & $\$ 4,775$ \\
\hline$\$ 100,000$ Above & 368 & $20 \%$ & $\$ 17,244$ & $\$ 3,329$ & $\$ 2,075$ & $\$ 4,956$ \\
\hline Not Reported & 287 & $15 \%$ & $\mathrm{n} / \mathrm{a}$ & $\$ 4,069$ & $\$ 1,173$ & $\$ 10$ \\
\hline TOTAL & 1,883 & $100 \%$ & $\$ 14,311^{*}$ & $\$ 730$ & $\$ 4,558$ & $\$ 3,876$ \\
\hline
\end{tabular}

Notes: This data only represents Fall and Spring financial aid data and is accurate as of March 31, 2016. Please note that small changes to Spring 2016 awards are possible before the data is finalized. Family Income Groups are based on the Total Family Income (including untaxed income) as reported on student FAFSA records. Full-time Students is a headcount based on at least 24 credit hours during Fall and Spring terms. Average Gift Aid includes all grants and scholarships from Federal, State, University and other private sources administered by the Financial Aid Office. Student waivers are also included in the Gift Aid amount. Gift Aid does not include the parental contribution towards EFC. Net Cost of Attendance is the actual average of the total Costs of Attendance (which will vary by income group due to the diversity of students living on- \& off- campus) minus the average Gift Aid amount. Net Tuition \& Fees is the actual average of the total costs of tuition and fees (which will vary by income group due to the amount of credit hours students are enrolled) minus the average Gift Aid amount (see page 16 for list of fees that are included). Average Loan Amount includes Federal (Perkins, Stafford, Ford Direct, and PLUS loans) and all private loans. 'Not Reported' represents the students who did not file a FAFSA. The bottom-line Total/Average represents the average of all full-time undergraduate Florida residents (note*: the total Net Cost of Attendance does not include students who did not report their family income data. 


\section{UNIVERSITY REVENUES}

University Revenues (in Millions of Dollars)

EDUCATION \& GENERAL

Main Operations

State Funds

Tuition

Subtotal
2014-15

$\$ 24.3$

$\$ 19.3$

$\$ 43.6$
2015-16

$\$ 27.4$

$\$ 21.7$

$\$ 49.1$

\section{OTHER BUDGET ENTITIES}

$\begin{array}{lcc}\text { Auxiliary Enterprises } & \$ 12.3 & \$ 19.4 \\ \text { Contracts \& Grants } & \$ 2.9 & \$ 3.8 \\ \text { Local Funds } & \$ 3.8 & \$ 3.9 \\ \text { Faculty Practice Plans } & \$ 0 & \$ 0\end{array}$

Note: State funds include recurring and non-recurring General Revenue funds, Lottery funds appropriated by the Florida Legislature. Actual tuition includes base tuition and tuition differential fee revenues for resident and nonresident undergraduate and graduate students net of waivers. Source: Tables $1 \mathrm{~A} \& 1 \mathrm{E}$ of the annual Accountability Report. 


\section{UNIVERSITY TUITION, FEES AND HOUSING PROJECTIONS}

\begin{tabular}{|c|c|c|c|c|c|c|c|}
\hline \multicolumn{8}{|l|}{ University of South Florida St. Petersburg } \\
\hline \multirow[t]{2}{*}{ Undergraduate Students } & \multicolumn{3}{|c|}{ 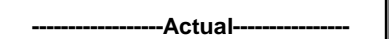 } & \multicolumn{4}{|c|}{ 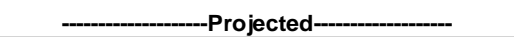 } \\
\hline & 2013-14 & 2014-15 & 2015-16 & 2016-17 & 2017-18 & 2018-19 & 2019-20 \\
\hline \multicolumn{8}{|l|}{ Tuition: } \\
\hline Base Tuition - (0\% inc. for $2016-17$ to $2019-20)$ & $\$ 105.07$ & $\$ 105.07$ & $\$ 105.07$ & $\$ 105.07$ & $\$ 105.07$ & $\$ 105.07$ & $\$ 105.07$ \\
\hline Tuition Differential $^{5}$ & 35.14 & $\$ 35.14$ & $\$ 35.14$ & $\$ 35.14$ & $\$ 35.14$ & $\$ 35.14$ & $\$ 35.14$ \\
\hline Total Base Tuition \& Differential per Credit Hour & $\$ 140.21$ & $\$ 140.21$ & $\$ 140.21$ & $\$ 140.21$ & $\$ 140.21$ & $\$ 140.21$ & $\$ 140.21$ \\
\hline \% Change & & $0.0 \%$ & $0.0 \%$ & $0.0 \%$ & $0.0 \%$ & $0.0 \%$ & $0.0 \%$ \\
\hline \multicolumn{8}{|l|}{ Fees (per credit hour): } \\
\hline$\overline{\text { Student Financial Aid }^{1}}$ & $\$ 5.25$ & $\$ 5.25$ & $\$ 5.25$ & $\$ 5.25$ & $\$ 5.25$ & $\$ 5.25$ & $\$ 5.25$ \\
\hline Capital Improvement $^{2}$ & $\$ 6.76$ & $\$ 6.76$ & $\$ 6.76$ & $\$ 6.76$ & $\$ 6.76$ & $\$ 6.76$ & $\$ 6.76$ \\
\hline Activity \& Service & $\$ 25.63$ & $\$ 25.63$ & $\$ 25.63$ & $\$ 25.63$ & $\$ 25.63$ & $\$ 25.63$ & $\$ 25.63$ \\
\hline Health & $\$ 4.90$ & $\$ 4.90$ & $\$ 4.90$ & $\$ 4.90$ & $\$ 4.90$ & $\$ 4.90$ & $\$ 4.90$ \\
\hline Athletic & $\$ 2.45$ & $\$ 2.45$ & $\$ 2.45$ & $\$ 2.45$ & $\$ 2.45$ & $\$ 2.45$ & $\$ 2.45$ \\
\hline Transportation Access & $\$ 2.25$ & $\$ 2.25$ & $\$ 2.25$ & $\$ 2.25$ & $\$ 2.25$ & $\$ 2.25$ & $\$ 2.25$ \\
\hline Technology ${ }^{1}$ & $\$ 5.25$ & $\$ 5.25$ & $\$ 5.25$ & $\$ 5.25$ & $\$ 5.25$ & $\$ 5.25$ & $\$ 5.25$ \\
\hline Green Fee (USF, NCF, UWF only) & $\$ 1.00$ & $\$ 1.00$ & $\$ 1.00$ & $\$ 1.00$ & $\$ 1.00$ & $\$ 1.00$ & $\$ 1.00$ \\
\hline \multicolumn{8}{|l|}{ Student Life \& Services Fee (UNF only) } \\
\hline \multicolumn{8}{|l|}{ Marshall Center Fee (USF only) } \\
\hline \multicolumn{8}{|l|}{ Student Affairs Facility Use Fee (FSU only) } \\
\hline Total Fees & $\$ 53.49$ & $\$ 53.49$ & $\$ 53.49$ & $\$ 53.49$ & $\$ 53.49$ & $\$ 53.49$ & $\$ 53.49$ \\
\hline Total Tuition and Fees per Credit Hour & $\$ 193.70$ & $\$ 193.70$ & $\$ 193.70$ & $\$ 193.70$ & $\$ 193.70$ & $\$ 193.70$ & $\$ 193.70$ \\
\hline$\%$ Change & & $0.0 \%$ & $0.0 \%$ & $0.0 \%$ & $0.0 \%$ & $0.0 \%$ & $0.0 \%$ \\
\hline \multicolumn{8}{|l|}{ Fees (block per term): } \\
\hline \multicolumn{8}{|l|}{$\overline{\text { Activity \& Service }}$} \\
\hline \multicolumn{8}{|l|}{ Health } \\
\hline Athletic & $\$ 5.00$ & $\$ 5.00$ & $\$ 5.00$ & $\$ 5.00$ & $\$ 5.00$ & $\$ 5.00$ & $\$ 5.00$ \\
\hline \multicolumn{8}{|l|}{ Transportation Access } \\
\hline \multicolumn{8}{|l|}{ Marshall Center Fee (USF only) } \\
\hline \multicolumn{8}{|l|}{ Student Affairs Facility Use Fee (FSU only) } \\
\hline \multicolumn{8}{|l|}{ List any new fee proposed } \\
\hline Total Block Fees per term & $\$ 5.00$ & $\$ 5.00$ & $\$ 5.00$ & $\$ 5.00$ & $\$ 5.00$ & $\$ 5.00$ & $\$ 5.00$ \\
\hline$\%$ Change & & $0.0 \%$ & $0.0 \%$ & $0.0 \%$ & $0.0 \%$ & $0.0 \%$ & $0.0 \%$ \\
\hline Total Tuition for 30 Credit Hours & $\$ 4,206.30$ & $\$ 4,206.30$ & $\$ 4,206.30$ & $\$ 4,206.30$ & $\$ 4,206.30$ & $\$ 4,206.30$ & $\$ 4,206.30$ \\
\hline Total Fees for 30 Credit Hours & $\$ 1,614.70$ & $\$ 1,614.70$ & $\$ 1,614.70$ & $\$ 1,614.70$ & $\$ 1,614.70$ & $\$ 1,614.70$ & $\$ 1,614.70$ \\
\hline Total Tuition and Fees for 30 Credit Hours & $\$ 5,821.00$ & $\$ 5,821.00$ & $\$ 5,821.00$ & $\$ 5,821.00$ & $\$ 5,821.00$ & $\$ 5,821.00$ & $\$ 5,821.00$ \\
\hline \$ Change & & $\$ 0.00$ & $\$ 0.00$ & $\$ 0.00$ & $\$ 0.00$ & $\$ 0.00$ & $\$ 0.00$ \\
\hline$\%$ Change & & $0.0 \%$ & $0.0 \%$ & $0.0 \%$ & $0.0 \%$ & $0.0 \%$ & $0.0 \%$ \\
\hline \multicolumn{8}{|l|}{ Out-of-State Fees } \\
\hline$\overline{\text { Out-of-State Undergraduate Fee }}$ & $\$ 346.50$ & $\$ 346.50$ & $\$ 346.50$ & $\$ 346.50$ & $\$ 346.50$ & $\$ 346.50$ & $\$ 346.50$ \\
\hline Out-of-State Undergraduate Student Financial $\mathrm{Aid}^{3}$ & $\$ 17.32$ & $\$ 17.32$ & $\$ 17.32$ & $\$ 17.32$ & $\$ 17.32$ & $\$ 17.32$ & $\$ 17.32$ \\
\hline Total per credit hour & $\$ 363.82$ & $\$ 363.82$ & $\$ 363.82$ & $\$ 363.82$ & $\$ 363.82$ & $\$ 363.82$ & $\$ 363.82$ \\
\hline$\%$ Change & & $0.0 \%$ & $0.0 \%$ & $0.0 \%$ & $0.0 \%$ & $0.0 \%$ & $0.0 \%$ \\
\hline Total Tuition for 30 Credit Hours & $\$ 14,601.30$ & $\$ 14,601.30$ & $\$ 14,601.30$ & $\$ 14,601.30$ & $\$ 14,601.30$ & $\$ 14,601.30$ & $\$ 14,601.30$ \\
\hline Total Fees for 30 Credit Hours & $\$ 2,134.30$ & $\$ 2,134.30$ & $\$ 2,134.30$ & $\$ 2,134.30$ & $\$ 2,134.30$ & $\$ 2,134.30$ & $\$ 2,134.30$ \\
\hline Total Tuition and Fees for 30 Credit Hours & $\$ 16,735.60$ & $\$ 16,735.60$ & $\$ 16,735.60$ & $\$ 16,735.60$ & $\$ 16,735.60$ & $\$ 16,735.60$ & $\$ 16,735.60$ \\
\hline \$Change & & $\$ 0.00$ & $\$ 0.00$ & $\$ 0.00$ & $\$ 0.00$ & $\$ 0.00$ & $\$ 0.00$ \\
\hline$\%$ Change & & $0.0 \%$ & $0.0 \%$ & $0.0 \%$ & $0.0 \%$ & $0.0 \%$ & $0.0 \%$ \\
\hline Housing/Dining ${ }^{4}$ & $\$ 11,451.60$ & $\$ 11,451.60$ & $\$ 11,451.60$ & $\$ 11,451.60$ & $\$ 11,451.60$ & $\$ 11,451.60$ & $\$ 11,451.60$ \\
\hline \$Change & & $\$ 0.00$ & $\$ 0.00$ & $\$ 0.00$ & $\$ 0.00$ & $\$ 0.00$ & $\$ 0.00$ \\
\hline$\%$ Change & & $0.0 \%$ & $0.0 \%$ & $0.0 \%$ & $0.0 \%$ & $0.0 \%$ & $0.0 \%$ \\
\hline 2 as approved by the Board of Governors. & & & & & & & \\
\hline${ }^{3}$ can be no more than $5 \%$ of tuition and the out-of-state $f$ & & & & & & & \\
\hline${ }^{4}$ combine the most popular housing and dining plans pro & ded to student & & & & & & \\
\hline${ }^{5}$ report current tuition differential. Only UF or FSU can ref & ct potential in & ses & & & & & \\
\hline
\end{tabular}

Note: The University will continually assess the need for changes to tuition \& fees in support of student success and fiscal responsibility consistent with FL Statute 1009.24 


\section{DEFINITIONS}

\section{Performance Based Funding}

Percent of Bachelor's Graduates Enrolled or Employed (\$25,000+) in the U.S. One Year After Graduation

\section{Median Wages of Bachelor's Graduates Employed Full-time in Florida One Year After Graduation}

This metric is based on the percentage of a graduating class of bachelor's degree recipients who are enrolled or employed (earning at least $\$ 25,000$ ) somewhere in the United States. Students who do not have valid social security numbers and are not found enrolled are excluded. Note: This data now non-Florida employment data.

Sources: State University Database System (SUDS), Florida Education \& Training Placement Information Program (FETPIP) analysis of Wage Record Interchange System (WRIS2) and Federal Employment Data Exchange (FEDES), and National Student Clearinghouse (NSC).

This metric is based on annualized Unemployment Insurance (UI) wage data from the fourth fiscal quarter after graduation for bachelor's recipients. UI wage data does not include individuals who are self-employed, employed out of state, employed by the military or federal government, those without a valid social security number, or making less than minimum wage. Sources: State University Database System (SUDS), Florida Education \& Training Placement Information Program (FETPIP), National Student Clearinghouse.

For each of the last four years of data, the annual undergraduate total full expenditures (includes direct and indirect expenditures) were divided by the total fundable student credit hours to create a cost per credit hour for each year. This cost per credit hour was then multiplied by 30 credit hours to derive an average annual cost. The average annual cost for each of the four years was summed to provide an average cost per degree for a baccalaureate degree that requires 120 credit hours. Sources: State University Database System (SUDS), Expenditure Analysis: Report IV.

\section{Six Year FTIC Graduation Rate}

This metric is based on the percentage of first-time-in-college (FTIC) students who started in the Fall (or summer continuing to Fall) term and had graduated from the same institution within six years. Source: Accountability Report (Table 4D).

\section{Academic \\ Progress Rate \\ 2nd Year Retention \\ with GPA Above 2.0}

This metric is based on the percentage of first-time-in-college (FTIC) students who started in the Fall (or summer continuing to Fall) term and were enrolled full-time in their first semester and were still enrolled in the same institution during the Fall term following their first year with had a grade point average (GPA) of at least 2.0 at the end of their first year (Fall, Spring, Summer). Source: Accountability Report (Table 4B).
University Access Rate Percent of Undergraduates with a Pell-grant

\section{Bachelor's Degrees within Programs of Strategic Emphasis}

\section{Graduate Degrees within Programs of Strategic Emphasis}

This metric is based the number of undergraduates, enrolled during the fall term, who received a Pell-grant during the fall term. Unclassified students, who are not eligible for Pellgrants, were excluded from this metric. Source: Accountability Report (Table 3E).

This metric is based on the number of baccalaureate degrees awarded within the programs designated by the Board of Governors as 'Programs of Strategic Emphasis'. A student who has multiple majors in the subset of targeted Classification of Instruction Program codes will be counted twice (i.e., double-majors are included). Source: Accountability Report (Table $4 \mathrm{H})$.

This metric is based on the number of graduate degrees awarded within the programs designated by the Board of Governors as 'Programs of Strategic Emphasis'. A student who has multiple majors in the subset of targeted Classification of Instruction Program codes will be counted twice (i.e., double-majors are included). Source: Accountability Report (Table $5 C)$. 


\section{BOG Choice Metrics}

\section{Percent of Bachelor's Degrees Without Excess Hours}

This metric is based on the percentage of baccalaureate degrees awarded within $110 \%$ of the credit hours required for a degree based on the Board of Governors Academic Program Inventory.

Note: It is important to note that the statutory provisions of the "Excess Hour Surcharge" (1009.286, FS) have been modified several times by the Florida Legislature, resulting in a phased-in approach that has created three different cohorts of students with different requirements. The performance funding metric data is based on the latest statutory requirements that mandates $110 \%$ of required hours as the threshold. In accordance with statute, this metric excludes the following types of student credits (ie, accelerated mechanisms, remedial coursework, non-native credit hours that are not used toward the degree, non-native credit hours from failed, incomplete, withdrawn, or repeated courses, credit hours from internship programs, credit hours up to 10 foreign language credit hours, and credit hours earned in military science courses that are part of the Reserve Officers' Training Corps (ROTC) program). Source: State University Database System (SUDS).

\section{BOT Choice Metrics}

\section{Key Performance Indicators \\ Teaching \& Learning Metrics}

Freshmen in Top $10 \%$

of HS Graduating Class

Professional/Licensure Exam First-time Pass Rates

\section{Average Time to Degree}

Mean Years for FTIC

in $120 \mathrm{hr}$ programs

FTIC Graduation Rates

In 4 years (or less)

\section{Bachelor's Degrees Awarded}

Graduate Degrees Awarded

\section{Bachelor's Degrees Awarded To African-American and Hispanic Students}

Percent of all degree-seeking, first-time, first-year (freshman) students who had high school class rank within the top $10 \%$ of their graduating high school class. As reported by the university to the Common Data Set (C10).

The number of exams with first-time pass rates above and below the national or state average, as reported in the annual Accountability report, including: Nursing, Law, Medicine (3 subtests), Veterinary, Pharmacy, Dental (2 subtests), Physical Therapy, and Occupational Therapy.

This metric is the number of years between the start date (using date of most recent admission) and the end date (using the last month in the term degree was granted) for a graduating class of first-time, single-major baccalaureates in 120 credit hour programs within a (Summer, Fall, Spring) year.

As reported in the annual Accountability report (table 4D), First-time-in-college (FTIC) cohort is defined as undergraduates entering in fall term (or summer continuing to fall) with fewer than 12 hours earned since high school graduation. The rate is the percentage of the initial cohort that has either graduated from or is still enrolled in the same institution by the fourth academic year. Both full-time and part-time students are used in the calculation. The initial cohort is revised to remove students, who have allowable exclusions as defined by IPEDS, from the cohort.

This is a count of baccalaureate degrees awarded as reported in the annual Accountability Report (Table 4G).

This is a count of graduate degrees awarded as reported in the Accountability Report (Table $5 B)$.

Non-Hispanic Black and Hispanic do not include students classified as Non-Resident Alien or students with a missing race code - as reported in the Accountability Report (table 4I).

Students who earn two distinct degrees in the same term are counted twice - whether their degrees are from the same six-digit CIP code or different CIP codes. Students who earn only one degree are counted once - even if they completed multiple majors or tracks. Percentage of Degrees is based on the number of baccalaureate degrees awarded to non-Hispanic Black and Hispanic students divided by the total degrees awarded - excluding those awarded to non-resident aliens and unreported. 
Adult (Aged 25+)

Undergraduates Enrolled

\section{Percent of Undergraduate FTE Enrolled in Online Courses}

Percent of Bachelor's Degrees in STEM \& Health

Percent of Graduate Degrees in STEM \& Health
This metric is based on the age of the student at the time of enrollment (not upon entry). Age acts as a surrogate variable that captures a large, heterogeneous population of adult students who often have family and work responsibilities as well as other life circumstances that can interfere with successful completion of educational objectives.

Full-time Equivalent (FTE) student is a measure of instructional activity that is based on the number of credit hours that students enroll. FTE is based on the US definition, which divides undergraduate credit hours by 30 . Distance Learning is a course in which at least 80 percent of the direct instruction of the course is delivered using some form of technology when the student and instructor are separated by time or space, or both (per 1009.24(17), F.S.). The percentage of baccalaureate degrees that are classified as STEM by the Board of Governors in the SUS program inventory as reported in the annual Accountability Report (Table 4H).

The percentage of baccalaureate degrees that are classified as STEM by the Board of Governors in the SUS program inventory as reported in the annual Accountability Report (Table 5C). 


\section{Student Debt Summary}

\section{Percent of Bachelor's Recipients with Debt}

This is the percentage of bachelor's graduates in a given academic year who entered the university as a first-time-in-college (FTIC) student and who borrowed through any loan programs (institutional, state, Federal Perkins, Federal Stafford Subsidized and unsubsidized, private) that were certified by your institution - excludes parent loans. Source: Common Dataset (H4).

This is the average amount of cumulative principal borrowed (from any loan program certified by the institution) for each native, FTIC bachelor's recipient in a given academic year that graduated with debt - see metric definition above. This average does NOT include students who did not enter a loan program that was certified by the institution. Source: Common Dataset (H5).

Student loan cohort default rate (CDR) data includes undergraduate and graduate students, and refers to the three federal fiscal year period when the borrower enters repayment and ends on the second fiscal year following the fiscal year in which the borrower entered repayment. Cohort default rates are based on the number of borrowers who enter repayment, not the number and type of loans that enter repayment. A borrower with multiple loans from the same school whose loans enter repayment during the same cohort fiscal year will be included in the formula only once for that cohort fiscal year. Default rate debt includes: Federal Stafford Loans, and Direct Stafford/Ford Loans - for more information see: http://ifap.ed.gov/DefaultManagement/CDRGuideMaster.html.

\begin{tabular}{|c|c|c|c|}
\hline $\begin{array}{l}\text { Cohort } \\
\text { Fiscal } \\
\text { Year }\end{array}$ & $\begin{array}{c}\text { Year } \\
\text { Published }\end{array}$ & $\begin{array}{l}\text { Borrowers in the Numerator } \\
\text { Borrowers in the Denominator }\end{array}$ & $\begin{array}{l}\frac{\text { 3-Yr Time Period }}{\text { (Numerator) }} \\
\text { 1-Yr Time Period } \\
\text { (Denominator) }\end{array}$ \\
\hline 2009 & 2012 & $\begin{array}{l}\text { Borrowers who entered repayment in } 2009 \\
\text { and defaulted in } 2009,2010 \text { or } 2011 \\
\text { Borrowers who entered repayment in } 2009\end{array}$ & $\frac{10 / 01 / 2008 \text { to } 9 / 30 / 2011}{10 / 01 / 2008 \text { to } 9 / 30 / 2009}$ \\
\hline 2010 & 2013 & $\begin{array}{l}\text { Borrowers who entered repayment in } 2010 \\
\text { and defaulted in } 2010,2011 \text { or } 2012 \\
\text { Borrowers who entered repayment in } 2010\end{array}$ & $\frac{10 / 01 / 2009 \text { to } 9 / 30 / 2012}{10 / 01 / 2009 \text { to } 9 / 30 / 2010}$ \\
\hline 2011 & $2014 *$ & $\begin{array}{l}\text { Borrowers who entered repayment in } 2011 \\
\text { and defaulted in } 2011,2012 \text { or } 2013 \\
\text { Borrowers who entered repayment in } 2011\end{array}$ & $\frac{10 / 01 / 2010 \text { to } 9 / 30 / 2013}{10 / 01 / 2010 \text { to } 9 / 30 / 2011}$ \\
\hline 2012 & 2015 & $\begin{array}{l}\text { Borrowers who entered repayment in } 2012 \\
\text { and defaulted in } 2012,2013 \text { or } 2014 \\
\text { Borrowers who entered repayment in } 2012\end{array}$ & $\frac{10 / 01 / 2011 \text { to } 9 / 30 / 2014}{10 / 01 / 2011 \text { to } 9 / 30 / 2012}$ \\
\hline 2013 & 2016 & $\begin{array}{l}\text { Borrowers who entered repayment in } 2013 \\
\text { and defaulted in } 2013,2014 \text { or } 2015 \\
\text { Borrowers who entered repayment in } 2013\end{array}$ & $\frac{10 / 01 / 2012 \text { to } 9 / 30 / 2015}{10 / 01 / 2012 \text { to } 9 / 30 / 2013}$ \\
\hline 2014 & 2017 & $\begin{array}{l}\text { Borrowers who entered repayment in } 2014 \\
\text { and defaulted in } 2014,2015 \text { or } 2016 \\
\text { Borrowers who entered repayment in } 2014\end{array}$ & $\frac{10 / 01 / 2013 \text { to } 9 / 30 / 2016}{10 / 01 / 2013 \text { to } 9 / 30 / 2014}$ \\
\hline 2015 & 2018 & $\begin{array}{l}\text { Borrowers who entered repayment in } 2015 \\
\text { and defaulted in } 2015,2016 \text { or } 2017 \\
\text { Borrowers who entered repayment in } 2015\end{array}$ & $\frac{10 / 01 / 2014 \text { to } 9 / 30 / 2017}{10 / 01 / 2014 \text { to } 9 / 30 / 2015}$ \\
\hline
\end{tabular}

